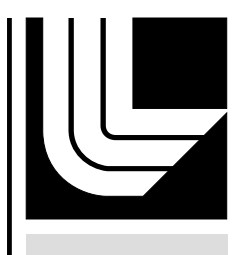

LAW RENCE LIVERMORE N A TION A L LABORATORY

\title{
History and Evolution of Control Banding: A Review
}

D. Zalk, D. Nelson

July 28, 2006

Journal of Occupational and Environmental Hygiene 
This document was prepared as an account of work sponsored by an agency of the United States Government. Neither the United States Government nor the University of California nor any of their employees, makes any warranty, express or implied, or assumes any legal liability or responsibility for the accuracy, completeness, or usefulness of any information, apparatus, product, or process disclosed, or represents that its use would not infringe privately owned rights. Reference herein to any specific commercial product, process, or service by trade name, trademark, manufacturer, or otherwise, does not necessarily constitute or imply its endorsement, recommendation, or favoring by the United States Government or the University of California. The views and opinions of authors expressed herein do not necessarily state or reflect those of the United States Government or the University of California, and shall not be used for advertising or product endorsement purposes. 


\title{
HISTORY AND EVOLUTION OF CONTROL BANDING: A REVIEW
}

\author{
David. M. Zalk ${ }^{1}$ and Deborah Imel Nelson ${ }^{2}$ \\ 1. Lawrence Livermore National Laboratory*, P.O. Box 808 L-871, Livermore, CA \\ 94551-0808 zalk1@,1lnl.gov \\ 2. Geological Society of America, P.O. Box 9140, Boulder, CO, 80301-9140, \\ dnelson@geosociety.org
}

Control Banding (CB) strategies offer simplified solutions for controlling worker exposures to constituents often encountered in the workplace. The original CB model was developed within the pharmaceutical industry; however, the modern movement involves models developed for non-experts to input hazard and exposure potential information for bulk chemical processes, receiving control advice as a result. The CB approach utilizes these models for the dissemination of qualitative and semi-quantitative risk assessment tools being developed to complement the traditional industrial hygiene model of air sampling and analysis. It is being applied and tested in small and medium size enterprises (SMEs) within developed countries and industrially developing countries; however, large enterprises (LEs) have also incorporated these strategies within chemical safety programs. Existing research of the components of the most available CB model, the Control of Substances Hazardous to Health (COSHH) Essentials, has shown that exposure bands do not always provide adequate margins of safety, that there is a high rate of under-control errors, that it works better with dusts than with vapors, that there is an inherent inaccuracy in estimating variability, and that when taken together the outcomes of this model may lead to potentially inappropriate workplace confidence in chemical exposure reduction in some operations. Alternatively, large-scale comparisons of industry exposure data to this CB model's outcomes have indicated more promising results with a high correlation seen internationally. With the accuracy of the toxicological ratings and hazard band classification currently in question, their proper reevaluation will be of great benefit to the reliability of existing and future $\mathrm{CB}$ models. The need for a more complete analysis of $\mathrm{CB}$ model components and, most importantly, a more comprehensive prospective research process remains and will be important in understanding implications of the model's overall effectiveness. Since the CB approach is now being used worldwide with an even broader implementation in progress, further research toward understanding its strengths and weaknesses will assist in its further refinement and confidence in its ongoing utility.

\section{INTRODUCTION}

A foundation of the modern movement for Control Banding (CB) strategies is derived from programs initiated in the United Kingdom (UK) by the Health and Safety Executive (HSE). The need to provide guidance and assistance to small and medium size enterprises (SMEs, which employ about $90 \%$ of the UK workforce ${ }^{1}$ ) in meeting requirements to conduct risk assessments of chemical exposures led to the HSE development of a program known as the Control of Substances Hazardous to Health (COSHH) Essentials. In 1998 the HSE published a series of papers outlining a CB strategy of creating a model in which the hazard was combined with the potential exposure to determine a recommended level of control approach. European Union (EU) risk phrases were used to rank the hazard of a chemical, and potential for exposure was estimated by the quantity in 
use, and the volatility of liquids or dustiness of solids. The scheme uses information associated with hazardous chemicals to develop hazard groups. These hazard groups are derived for a variety of chemicals and are designated by experienced toxicologists. When a hazard group associated with a chemical is selected by the manager of a Small- and Medium-Sized Enterprise (SME), toxicological expertise is utilized without the need for an on-site expert. This is an important foundation for the eventual consideration of the exposure potential to the chemical. The remainder of the decision making process includes the volume of chemical used, and likelihood of the chemical becoming airborne, estimated by the dustiness or volatility of the source compound. When these parameters are entered into a work sheet, the suggested control approach is identified. The end product is the selection of a control guidance sheet with both general and specific advice for common tasks ${ }^{2}$.

In the development of the CB model, Maidment ${ }^{3}$ stressed the importance of limiting the number of factors in the model to reduce its complexity and increase its applicability for non-experts. Although in theory there can be a stratification of risk across many levels, each additional level leads to a more intricate tool for the SME manager, which as an end product may hamper its overall intended utility. To achieve this balance of simplicity and effectiveness Maidment suggested four categories, or "bands", to assist in preventing exposure to chemicals. These four control strategies are a grouping of three levels of engineering containment based on sound industrial hygiene (IH) principles, with professional IH expertise as a fourth category. Within this model, these generic control strategies have also been adapted to address chemical exposure potential where the control guidance sheet (CGS) approaches may not be appropriate or practical. These other $\mathrm{CB}$ strategies utilize the banding approach to assist in directly assigning personal protection equipment (PPE) such as an appropriate level of respiratory protection and addressing dermal exposure potential ${ }^{1}$.

In a historical context, the banding of risk began in the 1970s and 1980s relating to explosive events, radiation, lasers and biological agents. The pharmaceutical industry should be credited with the initiation of exposure control categorization utilizing an industrial hygiene basis ${ }^{4,5}$ with its work in the late 1980s and early 1990s. During this period approaches to protect workers handling products with limited pharmacological and toxicological data led to efforts to stratify toxicological hazards and link them directly to simplified, commensurate control strategies during the production phases of product development ${ }^{6,7}$. These control approaches for pharmacological agent exposures were divided into five hazard categories ${ }^{5}$. This effort to address the growing potency of newly developed compounds followed the path of the microbiological and biomedical industries controlling exposures to increasingly toxic microorganisms within the four categories of the Biosafety Level approach ${ }^{8}$. Formally, the establishment of in-house Occupational Exposure Bands (OEBs) by the Association of the British Pharmaceutical Industry $(\mathrm{ABPI})^{9}$ assisted the product development phase of the industry to achieve a method for compliance with the COSHH regulations in a manner later adapted to the COSHH Essentials to address chemical exposures. There were several forces beyond the regulatory realm that also led to the $\mathrm{CB}$ model's adaptation and expansion into the chemical arena. Perhaps the most significant was the recognition that the traditional process of establishing occupational exposure limits (OELs), against which measurements of airborne concentrations of chemicals could be compared to ensure that exposures are controlled, was quickly losing ground by orders of magnitude to the increasing number of chemicals posing a threat to worker health ${ }^{1}$. Forces that drive the evolution of the CB model continue to this day. The nanotechnology industry is seeing 
itself akin to pharmaceutical and microbiological industries in that they are facing similar limitations in toxicological data. A CB model that addresses exposure to nanoparticlulate has recently been presented in concept as a practical approach to achieve exposure control in the absence of this data ${ }^{10}$.

\section{REVIEW OF CB LITERATURE}

The peer-reviewed literature on $\mathrm{CB}$ approaches (mostly relative to COSHH Essentials) can be summarized according to the development of the models, the use of databases to support the models, and the models' validation. CB has its roots in a number of qualitative $^{11}$ and semi-quantitative ${ }^{12}$ risk assessment approaches which began to appear in the 1970s and evolving in the 1980s relating the assessment of catastrophic failure probabilities at large chemical facilities ${ }^{13}$. An example of this is a risk matrix describing the likelihood and probable severity of an event, e.g. an explosion or release of toxic material, developed for use by a large chemical enterprise. As Money ${ }^{13}$ presented, there are a number of relevant strategies that were borrowed from and built upon during previous efforts and it is not always possible to trace the steps by relying on chronological appearance in the peer-reviewed literature. What is evident is that there was much exchange of information and ideas amongst occupational health practitioners and scientists in the chemical, biological, and pharmaceutical industries during that period of time $e^{1,5,14}$.

\section{Model development; linking toxicology to control}

In an early, perhaps the first, published report in which toxicological data were linked directly to an appropriate level of control. Money ${ }^{14}$ presented a structured approach to design and operation of a chemical plant that handles aromatic amines, nitro compounds, and equivalent agents with carcinogenic potential based on a carcinogenic ranking system. This was a broad approach for ensuring that appropriate measures would be in place to control risks from these chemicals from both routine and abnormal operations. It was truly simple in that it utilized a basic exposure scenario where the only determinant of exposure was the veracity of the toxicological data. Money suggested that this approach, which covered both inhalation and skin contact, should be applicable to similar approaches ranking relative hazards of chemicals ${ }^{15,16,17}$

This toxicology-to-control approach described by Money ${ }^{14}$ began by using four categories of toxicological outcome relating to carcinogenic potential, collapsed from a system utilizing six ${ }^{18}$ that considers both carcinogenic potency and weight of evidence. Money argued that while it is important to distinguish the potencies of different substances, in reality such a separation is artificial and impractical. Linearly matched with these four levels of carcinogenic potency were four levels of controls, progressing in complexity and stringency. Putting them together, these toxicology-to-control levels are then summarized as: (1) for all chemicals, use good basic IH; (2) for suspected animal carcinogens, increase to isolation of moderate exposure potential; (3) for suspected human carcinogens with moderate exposure potential increase, to containment and regular audits; and (4) for proven carcinogens with low exposure potential, increase to automated bulk transfers and process control.

The toxicology-to-control model was also applied by Nauman et al in $1996^{5}$ to exposures to pharmaceutical active ingredients in laboratory and manufacturing operations. The pharmaceutical industry had traditionally used risk assessment methods to establish OELs 
for active ingredients; however, the increasing potency of these agents led to a new approach based on the Biosafety Level concepts used in laboratories. Substantiated by a large database of air monitoring data for various operations they were able to distinguish five hazard categories (or performance-based exposure control limits, PB-ECL), based on toxicological and pharmacological properties of these agents and on the engineering controls and administrative procedures known to be effective in controlling exposure levels.

The Chemical Industries Association (CIA) further addressed toxicological information for chemical agents in their guidelines for safe handling of colorants (second version) ${ }^{19}$. In this document, inputs of hazard categorization (1-4), hazard classification (e.g., toxic, corrosive), associated risk phrase, and guideline control level (8-hour TWA) were linked to control recommendations for each hazard category. As both the CIA guidelines and the $\mathrm{COSHH}$ regulations were created in the UK, an ongoing discussion of chemical agent models began to develop. According to Guest ${ }^{20}$ the advice of the COSHH Approved Code of Practice, i.e., to set a self-imposed working standard for chemicals which did not have an official OEL, could not be followed by industry or government, due to the technical complexity of establishing OELs, the lack of adequate toxicological databases and experts, and the sheer volume of substances covered in the European Inventory of Existing Substances (EINECS) ${ }^{21}$. These factors led the CIA to develop chemical categorization guidelines for their member organizations.

Building on the earlier CIA guidance (1993) ${ }^{19}$ and the work of Gardner and Oldershaw $(1991)^{16}$, the later CIA guidelines $(1997)^{22}$ incorporated the Chemical Hazardous Information and Packaging (CHIP) Risk Phrases and guideline control levels, in addition to data on adverse effects in humans. The purpose of these guidelines was to provide a simple, broad-based, integrated approach for use by CIA members in classifying hazards. The categories were to be called OEBs and would only be developed when there were no other in-house, national, or international OELs. They would define the upper limit of acceptable exposure. As the number of control strategies is usually limited to approximately four levels, this approach was designed to cover 6 orders of magnitude, plus a special category. The upper limits (OEB C for dusts, OEB D for gases / vapors) were designed to "reflect good occupational hygiene practice" and the maximum dust concentration in the COSHH regulations $\left(10 \mathrm{mg} / \mathrm{m}^{3}\right)$.

\section{Model development; the exposure prediction step}

At this juncture, no one had yet factored the probability of exposure into the risk assessment and risk management aspects of a CB model. Although it had not yet been incorporated into the equation, much work was being conducted during the 1990s on predicting exposures. For example, Burstyn and Teschke's ${ }^{23}$ review on the methods of studying the determinants of exposure included work tasks, equipment used, environmental conditions, and existing controls. In evaluating the risk, a dedicated exposure model was used that is based on Cherrie and Schneider $^{24}$ by providing subjective exposure assessment using a structured approach based on descriptive workplace activities and environment. Using this model, subjective exposure assessment showed significant correlation with exposure measurements across 63 jobs and four different agents (asbestos, toluene, mixed respirable dust, and man-made mineral fibers). This serves as an excellent example of how dissecting existing models can lead to criteria to be used in developing other exposure control models and future toolkits. 
In studies of determinants of exposure reviewed by Burstyn and Teschke ${ }^{23}$, there was little attention devoted to volume of product used, and less to the physical characteristics of chemicals in use. The HSE played a pivotal role in developing a regulatory approach based on these concepts used to date ${ }^{25,26}$. While the work of the HSE was based in large part on that of the UK CIA ${ }^{20,22}$, which categorized substances into OEBs, it is apparent from the preceding discussion that many other groups have contributed to the development of COSHH Essentials. The challenge facing the HSE was to develop guidance which was practical for SMEs, used available hazard information, was easy to use and understand, and which relied upon readily available information (see Table I). These goals can be realized by using European risk phrases (R-phrases) and simple predictors of exposure to conduct a generic risk assessment, which leads to straightforward recommendations on risk management, i.e., control approaches.

Table I. Factors used in HSE's core model ${ }^{25}$.

\begin{tabular}{|l|l|l|l|}
\hline $\begin{array}{l}\text { HEALTH } \\
\text { HAZARD }\end{array}$ & $\begin{array}{l}\text { + EXPOSURE } \\
\text { POTENTIAL }\end{array}$ & \multicolumn{1}{|c|}{$\begin{array}{c}\text { ASENERIC RISK } \\
\text { ASSSSMENT }\end{array}$} & $\begin{array}{r}\rightarrow \text { CONTROL } \\
\text { APPROACH }\end{array}$ \\
\hline $\begin{array}{l}\text { Substances } \\
\text { allocated to a a } \\
\text { hazard band } \\
\text { using R-phrases }\end{array}$ & $\begin{array}{l}\text { Substances } \\
\text { allocated to a } \\
\text { dustiness or } \\
\text { volatility band } \\
\text { and a band for the } \\
\text { scale of use }\end{array}$ & $\begin{array}{l}\text { Combination of health } \\
\text { hazard and exposure } \\
\text { potential factors } \\
\text { determine desired level } \\
\text { of control }\end{array}$ & $\begin{array}{l}\text { Type of approach } \\
\text { needed to achieve } \\
\text { adequate control }\end{array}$ \\
\end{tabular}

The COSHH Essentials approach, as it later came to be known, builds on earlier approaches $5,14,16,22,27,28$. It also offers two other significant advances: it is specifically developed for SMEs and it includes control advice. The key components of the model include the hazard banding, exposure potential, and control approaches. Hazard banding is described more fully below ${ }^{26}$. It is important to point out, however, that from a British perspective, COSHH Essentials is limited to substances classified under CHIP, thereby excluding, e.g., pesticides and pharmaceuticals, which are outside the scope of those regulations, and also process-generated hazards such as wood dust, silica dust, and welding fumes. Exposure banding is a function of physical properties leading to likeliness for the material to become airborne (volatility of liquids or dustiness of solids, and the quantity in use $)^{3}$. These elements are combined to determine the appropriate control approach (see Table II). Therefore, there is perhaps a stronger link in the modern evolution of the CB model to the work of Burstyn ${ }^{23}$ and Cherrie ${ }^{24}$ than to the earlier toxicology-to-control approaches. Later versions of COSHH include PPE Essentials, offering advice for gloves and respirators, and for addressing dermal risks. Another feature of the COSHH Essentials web site is the newer Direct Advice topics for accessing hazard guidance by specific tasks, services, and processes (e.g., foundries, woodworking, beauty treatments, pubs, clubs and restaurants).

Table II. Control approaches used in COSHH Essentials ${ }^{25}$.

Control approach 1 - General ventilation. Good standard of general ventilation and good working practices.

Control approach 2 - Engineering control. Ranging from local exhaust ventilation to ventilated partial enclosure.

Control approach 3 - Containment. Containment or enclosure, allowing for limited, small scale breaches of containments.

Control approach 4 - Special. Seek expert advice. 
The developers felt that operation-based control guidance sheets (CGS) would provide the best format for advising SMEs. The approximately $100 \mathrm{CGS}^{29}$ now available are structured according to a standard format. This format contains sections on: design and equipment, maintenance, examination and testing, cleaning and housekeeping, PPE, training, supervision, a short list of references, a sample schematic of an engineering control, and an employee checklist for proper utilization of controls. Russell et al. ${ }^{25}$ states that use of the scheme will not in itself constitute a suitable and sufficient workplace risk assessment; it must therefore be considered as guidance and not a replacement for traditional IH. Employers should still consider other factors in their risk assessments, such as the need for health surveillance and the need to monitor exposure to ensure adequacy and suitability of controls. Similarly, it was pointed out that an over-protective approach would lack credibility, and deter promotion efforts and implementation, whereas an under-protective approach would not protect workers. Weighing these factors, it was generally agreed in the model development that a conservative approach would be the most responsible.

Brooke ${ }^{26}$ outlined three criteria for the toxicological basis of the UK approach: (1) simple and transparent, (2) make best use of available hazard information, and (3) recommend control strategies that vary according to degree of health hazard. The Rphrases that are agreed to throughout the EU facilitated these criteria, as they address all relevant toxicological endpoints. This idea had been proposed previously be Gardner and Oldershaw $^{16}$ and had formed the basis of similar strategies ${ }^{9,22,27}$. Brooke noted differences between these approaches and that of the HSE. COSHH Essential includes alignment between dust and vapor target exposure ranges and dose level cut-off values and is based on achievement of exposure levels anywhere in the target range, whereas the CIA recommends that exposures should be maintained "as low as reasonably practicable" ${ }^{, 20,22}$. Brooke's article achieved two goals: first, it explained the assignment of R-phrases to the Hazard Bands A-E utilized in the COSHH Essentials; and second, it compared these assignments to health-based OELs. The hazard bands, which are based on toxicological considerations, each divided by an order of magnitude in concentration range. As the relationship between the part per million (ppm) concentration of a vapor and the $\mathrm{mg} / \mathrm{m}^{3}$ concentration is a function of its molecular weight (and also temperature and pressure, though not discussed in this article), the working group which oversaw development of this approach decided to adopt a pragmatic approach and to align the exposure bands as seen in Table III below. Due to this alignment, "in $\mathrm{mg} / \mathrm{m}^{3}$ terms, the concentration range for substances in vapor form is substantially higher than that for the substance in particulate form, for the same toxicological hazard band."

Table III. Allocating R-phrases to hazard bands ${ }^{26}$.

\begin{tabular}{|l|l|l|}
\hline Hazard band & $\begin{array}{c}\text { Target airborne } \\
\text { concentration range } \\
\text { (Note 1) }\end{array}$ & \multicolumn{1}{|c|}{ R-phrases } \\
\hline $\mathrm{A}$ & $>1-10 \mathrm{mg} / \mathrm{m}^{3}$ dust; \\
& $>50-500 \mathrm{ppm}$ vapor & $\begin{array}{l}\mathrm{R} 36, \mathrm{R} 38, \text { all dusts and vapors not } \\
\text { allocated to another band (Note 2) }\end{array}$ \\
\hline $\mathrm{B}$ & $>0.1-1 \mathrm{mg} / \mathrm{m}^{3}$ dust; & $\mathrm{R} 20 / 21 / 22, \mathrm{R} 40 / 20 / 21 / 22$ \\
& $>5-50 \mathrm{ppm} \mathrm{vapor}$ & \\
\hline $\mathrm{C}$ & $>0.01-0.1 \mathrm{mg} / \mathrm{m}^{3}$ dust; & $\mathrm{R} 48 / 20 / 21 / 22, \mathrm{R} 23 / 24 / 25, \mathrm{R} 34$, \\
& $>0.5-5 \mathrm{ppm} \mathrm{vapor}$ & $\mathrm{R} 35, \mathrm{R} 37, \mathrm{R} 39 / 23 / 24 / 25, \mathrm{R} 41, \mathrm{R} 43$ \\
\hline $\mathrm{D}$ & $<0.01 \mathrm{mg} / \mathrm{m}^{3}$ dust; & $\mathrm{R} 48 / 23 / 24 / 25, \mathrm{R} 26 / 27 / 28$, \\
\hline
\end{tabular}




\begin{tabular}{|l|l|l|}
\hline & $<0.5$ ppm vapor & $\begin{array}{l}\text { R39/26/27/28, R40 Carc. Cat. 3, } \\
\text { R60, R61, R62, R63 }\end{array}$ \\
\hline E & See specialist advice & $\begin{array}{l}\text { R40 Muta. Cat. 3, R42, R45, R46, } \\
\text { R49 }\end{array}$ \\
\hline S: skin and eye contact & $\begin{array}{l}\text { Prevention or } \\
\text { reduction of skin } \\
\text { and/or eye exposure }\end{array}$ & $\begin{array}{l}\text { R34, R35, R36, R38, R41, R43, Sk } \\
\text { (Note 3) }\end{array}$ \\
\hline
\end{tabular}

In writing about the development of the model, Maidment ${ }^{3}$ stressed the importance of limiting the number of factors in the model to control its complexity and applicability. This simplicity was to be balanced with the hazard and exposure potential parameters necessary to predict an adequate control strategy. Toward this end, control strategies were collapsed into four main categories (Table II). Since characteristics of exposure potential can be summarized as those related to physical properties and those related to substance handling, Maidment focused on the dustiness of solids, and the volatility of liquids. The study indicated that three dustiness bands would adequately describe the properties of dusts and maintain the simplicity of the model: low, medium, and high. For liquids, the volatility of a liquid would be captured by consulting a graph of boiling point versus operating temperature, separated into three regions: low, medium, and high volatility. As a subsequent characteristic of operational factors the scale of the operation was classified as small-scale, medium-scale, and large-scale.

With these three articles ${ }^{3,25,26}$ the wider occupational safety and health community was thus introduced to the basics of the COSHH Essentials approach. While this strategy leans heavily on the work of historical models and approaches, it has a number of unique features, including an electronic version accessible via the internet. It meets all six of Money's ${ }^{13}$ core principles (understandability, availability, practicality, user-friendliness, confidence on the part of users, and transparent, consistent output). While welcoming the move by HSE to provide guidance in the form of CGS, Hudspith and Hay ${ }^{30}$ pointed out an additional obstacle to worker protection: communications barriers within companies. They recommended that HSE continue to stress the value of workforce involvement in health and safety issues. Despite its attributes, however, the COSHH Essentials model is subject to a number of limitations relative to the development of the model, development of databases, use of the model, and its validation and verification.

\section{Validation and Verification}

For purposes of this paper, validation focuses on the establishment of the soundness of a given model, whereas verification requires the evidence necessary to confirm its effectiveness. While it would be useful to validate a variety of the $\mathrm{CB}$ strategies proposed, only COSHH Essentials has been developed and implemented to the point that it has been the subject of almost all validation efforts. Also receiving attention is the International Labor Organization (ILO) Chemical Control Toolkit (ILO Toolkit), produced in collaboration with the HSE and the International Occupational Hygiene Association (IOHA). The ILO Toolkit is based on the HSE COSHH Essentials and is adapted for use worldwide ${ }^{31}$. For validation purposes, three aspects of model evaluation were applied by $\operatorname{Tischer}^{32}$ to COSHH Essentials. These aspects to validate the model include: internal (conceptual) validation of the model's assumptions and structure, external (performance) validation of the model predictions corresponding to professional IH monitoring data, and operational analysis of the understanding and implementation of the model's outcomes respective to its target group. 
However, before presenting these model aspects there are still many questions to be answered in all three categories. Kromhout ${ }^{33}$ took strong exception to the lack of exposure monitoring in "generic risk assessment tools like COSHH essentials and expert systems like the Estimation and Assessment of Substances Exposure (EASE)..." as they "... are known to be inaccurate and they do not take into account the various components of variability in exposure levels...". Kromhout built a strong case, estimating the variability in an eight-hour shift to be between 3 and 4000 fold, and delineating the sources of variability as spatial, between workers, and between groups. He argued that while providing exposure controls without having measured exposure concentrations would save money in the short term, in the long run it would be "penny wise but pound foolish".

Topping $^{34}$ responded that these arguments ignored the range of competencies in the workplace, and the number of firms handling chemicals however, he concurred that the use of "quality exposure data is extremely valuable for assessing the effectiveness of control measures". He did not directly address Kromhout's variability concern, but instead relied on the premise that COSHH Essentials is not intended to replace monitoring, but rather to provide needed help to SMEs. Topping pointed out that the cost of conducting the extensive monitoring suggested by Kromhout would be "astronomical" and that the capacity to do so does not exist. He allowed that the COSHH Essentials were designed to "err on the side of caution," that the strategy had been peer reviewed by the British Occupational Hygiene Society (BOHS) experts, and that there had been no complaints about the recommended controls being too stringent without addressing the lack of research to show that the controls have even been put into place, let alone that they have been verified to achieve the intended exposure control. Kromhout ${ }^{35}$ replied that he and the editor of the Annals of Occupational Hygiene questioned the role of tools like COSHH Essentials in the "collapse of full time training of occupational hygiene professionals in Britain through lack of demand for expertise." Kromhout's strongest criticism was that EASE and COSHH Essentials had not been properly evaluated prior to release, and that peer review by BOHS experts could not replace the rigorous evaluation of testing for reproducibility, validity, and peer review of results in the scientific literature. It was recommended that COSHH and EASE be used in the initial screening process. According to Maidment ${ }^{3}$, the core model was validated by predicted dust and vapor exposure ranges, and their corresponding three-tiered hierarchy of engineering controls with measured data, and by extensive peer review of the logic and content by experts. He noted that it was extremely difficult to find quality data for comparisons, and further, that the information describing control strategies often seemed to indicate that several control strategies were in use. Limited comparisons were described in his manuscript; heavy reliance was placed on peer review during the model's development and validation and specifically involved the HSE Advisory Committee on Toxic Substances (including Guest, Brooke and Money) and experts of the BOHS ${ }^{3,36}$. When taken as a whole, Topping did not address Kromhout's concerns of this unpublished peer review process. Therefore, not addressed are the potential weaknesses that one might find in the scientific literature when internal and external validation of the model is performed.

Brooke's ${ }^{26}$ work in comparing the R-phrases and resulting target airborne concentrations to the relevant health-based OELs on national lists (UK and German Maximum Allowable Concentrations (MAK) began to address the first category on internal validation for the COSHH Essentials. The work of Jones and Nicas ${ }^{31}$ reported below looked at both internal validation of the ILO Toolkit as compared to the UK HSE model 
and the external validation of the COSHH Essentials. The work of Tischer et al. ${ }^{32}$ and Maidment ${ }^{3}$ focused on the external validation and began to answer some of the questions relating to performance validation. A glaring weakness in the research at this time is present regarding the operational analysis of the given $\mathrm{CB}$ models.

Brooke was the first to identify the inherent difficulty in assigning dusts and vapors to equivalent bands designated elegantly by orders of magnitude (Table III). Resulting from this alignment of the bands, dusts have a higher margin of safety than vapors, especially for repeated exposure toxicity based R-phrases. Emphasizing the generic nature of this $\mathrm{CB}$ model and its provision for "adequate control" Brooke concluded that the margins offer "considerable reassurance" for vapors and "even greater reassurance" when used for dusts. Much of the model's weakness in this regard was balanced against the intended non-expert SME end-user with no risk assessment background. With this in mind Brooke explicitly noted that the model used in practice would require "continued evaluation of the allocation of the R-phrases to the hazard bands, such that the scheme may be revised and improved in the light of practical experience." 26 Brooke also reported that some categories of materials were arbitrarily assigned to a higher hazard category based on their toxicity characteristics, and this would provide an extra factor of 10 . It must be pointed out that the Hazard Band values are generally in the same order of magnitude as OELs (see Table III) and also that it is not uncommon for acceptable risk levels of OELs, which are based on a 40-hour work week that accounts for worker recovery periods, to be in the range of $10^{-4}$ to $10^{-3}$. In contrast, acceptable risk values in environmental settings, which are based on continuous, involuntary exposure (168 hours per week) of all members of the population with no recovery period ${ }^{38}$, are in the range of $10^{-6}$ to $10^{-5}$. Without understanding the basis of these underlying risk parameters, the problem then lies more in the lack of overall acceptance of higher-risk levels for occupational settings as compared to environmental settings. Solving this issue will require an improved communication of the reasons behind this risk differential and, therefore, a greater understanding of risk acceptability in occupational settings.

Jones and Nicas ${ }^{31,37}$ reported less positive results in their evaluation of the ILO Toolkit. The ILO Toolkit, as discussed above, was based on the COSHH Essentials strategy, but may not have been subject to the same periodic updates and revisions. They concluded that the calculation of safety margins (No Observed Adverse Effect Level (NOAEL), or the Lowest Observed Adverse Effect Level (LOAEL), divided by the high air concentration of the hazard band) resulted in values of $<100$ for Hazard Groups B and C, and $<250$ for Hazard D for vapors. They noted that these values should be in the range of 1000 to 10,000 for $\mathrm{R} 48 / 20$ (Danger of serious damage to health by prolonged (inhalation) exposure), depending on whether the NOAEL or LOAEL was utilized as the basis of calculation. That study made these calculations based on the generic COSHH criteria, to avoid any errors caused by incorrect assignments of hazard bands. A comparison of the R-phrases (taken from the HSE "Approved Supply List" (National Chemical Emergency Centre at http://www.the-ncec/cselite)) assigned to commonly-used solvents indicated that the hazard group ratings assigned by the ILO Toolkit were lower than seen in the COSHH Essentials, for 12 of 16 solvents. In 5 cases, the ILO Toolkit included an S notation (skin hazard) which was not on the R-phrases. Jones and Nicas ${ }^{31}$, 37 suggested that the authors of the ILO Toolkit should reconsider the hazard classification plan as the variations among CB strategies reduce trust on the part of users. Based on the small safety margins between doses that cause significant effect in animals and the exposure bands in the toolkits being evaluated, they also suggested target exposure levels be made available to end users. Without offering these to the user to 
evaluate whether exposures are in line with the minimal margin, a false sense of health protection in the workplace is permitted ${ }^{37}$.

Tischer ${ }^{32,39}$ and colleagues at the German Federal Institution for Industrial Safety and Medicine (BAuA) conducted the first and most complete external validation of the COSHH Essentials to date, based on independent measurement data. The primary empirical basis for their analysis was measurement data collected within the preceding decade during several BAuA field studies. Some data were also provided by the chemical industry. Tischer's team also set out to address the external validation of the COSHH Essentials exposure model. While stating that the accuracy of the model was represented by agreement between predicted and observed, they believed that statistical tests are not useful due to the uncertainties in empirical data such as variability, errors in measurements, or false or incomplete information. Due to a lack of available data for some professions, only those with more complete data sets were used in this study. There were apparently 958 data points available for evaluation: 732 for liquids, and 226 for solids.

The BAuA data were all obtained from their own laboratories, and all workplace measurements were conducted as per the German Technical Rules. Sampling durations were usually 1-4 hours, and were task-based, i.e., they corresponded to a specific scenario. Over 95\% were personal samples. Sources of uncertainty considered were volatility / dustiness, scale of use, and control strategy. For example, the uncertainty associated with volatility (of pure substances) was judged to be low, but quite complicated when mixtures were considered. Dustiness was considered to be a problem requiring additional attention. Scale of use was judged to be straightforward. (Most of the available data corresponded to the medium scale of use, with very little in the milliliter or tonne ranges.) Because of the limited quantity of data available, these researchers limited their analyses to scenarios in which the control strategy could be determined from the historical reports, generally matching one of the four control strategies. Comparisons of the predicted and actual data were conducted using frequency polygons overlaid with the range of predicted values and by calculating the percentage of the cases which were correctly or incorrectly predicted. Most of the data points fell within the predicted ranges. Per Balsat et al. ${ }^{12}$, Tischer $^{40}$ found that the $95^{\text {th }}$ percentile of data from different operations fit within the ranges predicted by the COSHH Essentials model. Exceptions were scenarios where some of the limited data points for solvent exposures were above the predicted range, such as in carpentry workshops and with adhesives applications where the chemical product are spread over a large surface area reflecting small-scale, dispersive operations. Exceedances also occurred in the handling of powdery substances in kilogram quantities under local exhaust ventilation.

Jones and Nicas ${ }^{41}$ also performed external validation by evaluating the ability of the COSHH Essentials to select an appropriate control approach and whether these controls achieved reduction of exposure concentrations. They compared reported air monitoring data and related use of ventilation systems, taken from the National Institute for Occupational Safety and Health (NIOSH) Health Hazard Evaluations (HHEs) for 31 vapor degreasing operations with 7 different solvents and 20 bag filling operations with 17 particulates (\#42). R-phrases for these liquids and dusts were obtained from the HSE National Chemical Emergency website (8 substances), the Australian "Approved Criteria for Classifying Hazardous Substances (2002) and the Hazardous Substances Data Base (HSDB) of the United States (US) National Library of Medicine (6 substances), and the Internet (9 substances). Volatility information was obtained from the HSDB, and 
dustiness and scale-of-use were obtained from the NIOSH HHEs. Using this information, Jones and Nicas determined the appropriate control approach, and compared the actual measured exposures to the maximum value of the exposure band of the recommended exposure band. This comparison resulted in two types of control errors: situations in which insufficient exposure control occurred in the presence of local exhaust ventilation (LEV) (under-control errors), and situations in which sufficient exposure control occurred in the absence of LEV (over-control errors). They found under-control errors in $96 \%$ of the 163 cases where LEV was present in vapor degreasing operations, and in 55\% of the 49 cases where LEV was present in bag filling operations ${ }^{4 \mathrm{P}}$.

Their findings led Jones and Nicas ${ }^{31,41}$ to multiple conclusions. They found that the exposure bands do not provide consistent, or adequate, margins of safety and the high rate of under-control errors highlighted the need to evaluate the effectiveness of installed LEV systems using capture efficiency and/or air monitoring techniques. The limited assignment of "dustiness" ratings to dusts complicates the model's process and indicates that specific guidance must be provided in cases where there is insufficient or inappropriate hazard information and that guidance on contacting professional assistance for engineering controls should be included on Task Guidance Sheets. Additionally, the R-phrase procedures, which include concentration "cut-off" values (e.g., the hazard classification would not be for a mixture with $<\mathrm{x} \%$ of the substance), are not compatible with US regulatory practice, which may result in measurements of the airborne concentrations of the constituents of a mixture, regardless of their percentage composition in the mixture.

Ruden and Hansson ${ }^{30}$ investigated the accuracy of the EU classifications for acute oral toxicity for 992 substances by comparing their acute toxicity categorization ("very toxic", "toxic", and "harmful") to the acute oral toxicity data available in RTECS ${ }^{\circledast}$ (Registry of Toxic Effects of Chemical Substances). Acute oral toxicity in rats is used because, although of minor importance for the complete toxicological profile, it offers a gauge of immediate toxicity with many substances lacking long-term data. They found that of the 992 substances that had enough data to undergo this evaluation, $15 \%$ (152) were assigned too low a danger class, and $8 \%$ (79) too high. Of those too low, or under-classified, 26 should be classified as "very toxic," 49 should be "toxic," and 77 should be "harmful". According to Ruden and Hansson, the EU classifications rules indicate that once a substance is placed into a category based on specific toxicological data, it cannot be downgraded to a lower category based on additional information. It is when different studies of appropriate scientific quality would lead to different categorizations that the rules are less clear. In this instance, the authors indicate that there is an "informal policy" in the EU to base its final classification on the most adverse outcome. A number of possibilities for this under-classification issue were noted, including variations in toxicity data from different laboratories; however, more issues arise relating to the EU informal policy. If the EU Commission has access to data not in $\operatorname{RTECS}^{\circledR}$, and these data support higher classifications, then the policy should default a substance categorization to a higher hazard class and not a lower one. Other possibilities exist such as the frequency of updating classifications, insufficient toxicity data searches, or problems with the RTECS ${ }^{\circledast}$ database. Regardless, it is difficult to accurately pinpoint a causal relationship as there is a "lack of transparency" in the Commission's classifications. For future classifications of substances the authors recommended the scientific basis be published to afford this transparency so when similar issues arise they can be addressed and rectified ${ }^{42}$. 
Although not always recognized as a validation parameter, the COSHH Essentials' CB model had ease-of-use and simplicity as intended design parameters for the non-expert end user ${ }^{3,26,29}$. Therefore, the results of an HSE survey to determine the utility of the COSHH Essentials should also be considered. A telephone survey was performed with 500 chemical purchasers who have used the older, paper version of COSHH Essentials ${ }^{43}$. The survey indicated that it had been utilized by $80 \%$, with only $5 \%$ finding it difficult to use and $95 \%$ willing to recommend it to other companies. In addition, $75 \%$ of those surveyed had taken action to control chemical exposures. Actions taken when utilizing the COSHH Essentials model included: chemical substitution (18\%), changing work procedures $(25 \%)$, changing the control measure used (36\%), providing information or training to workers $(48 \%)$, and checking existing control measures to ensure they are working (67\%).

\section{Variations of the chemical model}

Users of $\mathrm{CB}$ strategies quickly realized that one strategy would not fit all needs. Variations of the model and its use in practice have been developed by several nations including France, Germany, Belgium, The Netherlands, and Singapore and also by corporations. Interest in $\mathrm{CB}$ strategies on the part of the European occupational hygiene community was spurred by the introduction of the Chemical Agents Directive in $1998^{13}$, 44. Several approaches have resulted. The French approach ${ }^{45}$ evaluates the probable effectiveness of risk management in protecting workers at the company level. It suggests appropriate references to provide guidance based on the type of substance and handling procedures. In June 2007, a new European law on chemicals, REACH (Registration, Evaluation, Authorisation and Restriction of Chemicals), entered into force; at the same time, the European Chemicals Agency (ECHA) began operations. This law shifts greater responsibility to industry to manage the risks from chemicals and to provide safety information on the substances ${ }^{46}$. The European Chemical Industry Council (CEFIC) exposure management system (CEMAS) ${ }^{13,47}$ intends to provide a guidance tool for SME, to collect workplace exposure data which can be coupled with hazard information and deliver advice on risks and risk management, recommending whether exposure monitoring should be conducted.

The European Centre for Ecotoxicology and Toxicology of Chemicals (ECETOC) ${ }^{48}$ approach is a tiered and targeted risk assessment that could aid in the registration of large number of chemicals under REACH. This is a streamlined approach which applies CB concepts in a tiered manner with Tier 0 screening out chemicals not presenting an immediate risk to humans or the environment; Tier 1 identifying uses of a chemical which may present further risks, to be investigated in greater depth in Tier 2. In Tier 1, margins of exposure (MoE) are compared with generic OELs for the chemical's hazard category, while Tier 2 assessments are conducted in accordance with EU risk assessment principles. Toward that end, a database known as Solbase shows potential as a source from which CGSs could be developed. With partners from throughout Europe, Swuste et al. ${ }^{11}$, have tested Solbase, both for usability of the software, and suitability of the recommendations yielded by Solbase, using 535 new and existing solutions. Although most of these solutions currently relate to manual or material handling, noise and vibration, machine guarding, and other safety issues, few address air contaminants. The databank can be queried either by production process, or by hazard. 
Much of the literature for the evaluation and validation of CB models has been related to a concerted effort to create and drive a research agenda through workshops. This approach has been proven useful for developing earlier solutions-based programs beyond their national origin. Early solution-based initiatives include the noise control solutions from the UK $\mathrm{HSE}^{49}$, exposure reduction in mining from Australia ${ }^{50}$, and chemical substitution strategies from Denmark, the UK, the US, and The Netherlands to reduce health hazards ${ }^{51}$. A model for communication and evaluation of these programs began at the first IOHA Scientific Conference in 1992 with a workshop on sharing knowledge of preventive measures. This culminated in a $1994 \mathrm{WHO}$ experts meeting to stimulate the interchange of solutions toward the reduction of occupational risk and the formation of the Prevention and Control Exchange (PACE) working group ${ }^{52}$. This process has evolved into efforts such as the European Solbase, with many nations teaming together to develop a database of effective controls for workplace hazards and reduction of occupational risks $^{11}$. International CB workshops have been held in London (2002), Cincinnati (2004), and South Africa (2005). The workshops have led to an international agreement for coordinating the work of international agencies and their partners and a global implementation strategy for CB models. An example of this collaboration is the appropriate international forum that the workshops have provided for the Globally Harmonized System of Classification and Labeling of Chemicals (GHS). The GHS is a uniform, internationally developed, and standardized protocol for the toxicological basis for assigning chemicals to standardized hazard statements on labels and safety data sheets in manner that builds on the EU R-phrase process. From the beginning of the UK model's development it was made clear that when more data became available, chemical substances would need to be reclassified ${ }^{20}$. Should there be future reclassification efforts, it has been recommended that the scientific basis and decision matrix for these hazard classifications be standardized and readily available to achieve transparency for subsequent evaluations ${ }^{42}$. GHS is presented as a proper approach to build transparency to the process by including a core set of label elements to work towards harmonized hazard statements for each category and class of chemicals covered. It also has a harmonized approach to classifying mixtures of these chemicals. The GHS has also adopted the concept of the ILO Toolkit as part of its overall process to include exposure control approaches in parallel with its efforts for a chemical standardization process. While this may take some time to accomplish, it will eventually provide consistency of information on over 1500 commonly used chemicals and include a centralized procedure for frequent updates of information ${ }^{53}$.

Through these CB workshops, a process emphasizing the utility of available CB models has also led many countries to adapt and use them within their existing occupational risk management approaches. A two-stage risk assessment strategy (Regetox) was developed and tested in Belgium ${ }^{12,54,55}$ in response to the European Chemical Agents Directive $98 / 24 / \mathrm{EC}^{44}$, which requires companies to assess and manage chemical risks in the workplace. In order to minimize the number of chemicals (and resulting costs) for which risk assessment must be conducted, the first stage of the strategy utilizes the French "ranking of potential risk" based on R-phrase, annual quantity in use, and frequency of use, as described above ${ }^{56}$. Only products receiving a rating of medium or high are carried forward to the second stage, which utilizes the COSHH Essentials. When mixtures are being handled, the risks are evaluated for each harmful component according to the composition by weight of the mixture ${ }^{12}$. For cases in which contaminants are generated during the process, e.g. aerosols generated during spray painting, the EASE model is used. Feasibility studies conducted in two firms revealed lacking or inadequate MSDS. There was only one case in the two companies in which the strategy failed to reveal need 
for improvement in the work situation. The authors felt that simple examination of the work situation would have indicated the need for semi-quantitative risk assessment. Further lessons drawn from the trial are that most companies are not prepared to comply with the European Chemical Agents Directive, and that the use of the Regetox approach can be helpful to companies, but requires training of "prevention advisors" and a strategy to involve employers, staff members, and workers to assist in collecting basic information for the risk assessment ${ }^{56}$.

The Semi-Quantitative Risk Assessment (SQRA) developed in Singapore is intended to facilitate identification of chemical hazards, evaluation and potential for exposure, risk level determination, and prioritization of appropriate controls to address the identified risks. With the SQRA there are three methods for performing exposure evaluation that include personal exposure monitoring, using exposure factors and parameters, and/or applying empirical and theoretical formulas to estimate exposures at the plant or process design stage. The ILO Toolkit, which was renamed as the International Chemical Control Toolkit (ICCT) during the SQRA's development, was tested in parallel with applications of the SQRA to evaluate their utility and to perform comparisons based on theoretical and empirical aspects ${ }^{57}$. Direct comparison of the two approaches was stratified by their respective control approaches as compared to the SQRA risk level approach. The empirical comparison of the models uses actual personal air monitoring data used to derive the SQRA method's risk level to assess against the Toolkit's control approach. This comparison was performed on 27 selected SME processes including metal-working, paint manufacturing, chemical processing, printing, dry cleaning, and electronics industries. The results of the theoretical comparison indicate that the Toolkit and the SQRA method are somewhat consistent with any differences between the control approach and risk level being at most one to two bands. In the majority of cases using the empirical comparison it was determined that the Toolkit over-evaluates the risk relative to SQRA, leading to more conservative approaches relating to controls ${ }^{57}$.

Germany is the third largest chemical producer in the world, and the largest chemical exporter in the world ${ }^{58}$. As such, it has taken its responsibility to assist in sound management of chemicals in developing countries (Tischer and Scholaen 2003). Under its Convention Project on Chemical Safety, the technical arm of the German Development Agency's Society for Technical Cooperation (GTZ) has developed a Chemical Management Program Guide as part of its Pilot Project on Chemical Safety. The Chemical Management Guide is a method to demonstrate and document how chemical safety in emerging countries and small businesses can be improved and sustainability implemented in line with international standards. In more than 130 partner countries, GTZ is supporting 2,700 development projects and programs with the aim to improve the living conditions and perspectives of people in developing and transition countries. It has been implemented at international sites in Argentina, Indonesia, and EU countries. The GTZ chemical management guide and pilot project on chemical safety is a unique program developed specifically to meet the needs of small businesses and developing countries for addressing chemical hazards. A participatory training process is utilized to work to the selection of control technologies. The GTZ program acknowledges that $\mathrm{CB}$ models may be too sophisticated for many small enterprises in developing countries; medium- and larger enterprises often have more MSDS on site and therefore they have a greater potential for conducting risk assessments using the ILO Toolkit ${ }^{59}$.

Building upon the COSHH Essentials approach, countries have also begun to develop their own $\mathrm{CB}$ models to address national regulatory requirements and professional 
approaches. Stoffenmanager (www.Stoffenmanager.nl - accessed 14 October 2007) is a web-based software tool built for SMEs to assist in working safely with chemical substances. Their CB model factors in an exposure potential through the use of an interactive chemical risk management approach. This model approach was developed in The Netherlands to assist SMEs in assessing, prioritizing, and controlling risks associated with hazardous substances. The tool is based on the COSHH Essentials and a modified version of the Cherrie and Schneider ${ }^{24}$ inhalation exposure model ${ }^{60}$. Stoffenmanager is currently a generic tool that supports the inventory of the hazardous substances, assessing and controlling risks in a risk inventory, obtaining a plan for control measures, making instruction sheets for the workplace, and helping in storage according to guidelines. For the risk inventory, the employer uses R-phrases categorized according to COSHH Essentials. Then the employer completes an exposure assessment, involving response to 7 questions to determine the chemical's exposure class. The tool automatically calculates a risk score, a relative risk ranking. Thus an initial assessment of the health risk is completed. Using the tool's risk score, the employer can then calculate the efficacy of various control measures and choose the most effective ones ${ }^{61}$. The Stoffenmanager model has been recently evaluated utilizing targeted field surveys for many dust (i.e. animal feed, flour processing, textile, and construction) and liquid (i.e. solvents for metal, car body repair, and printing) industry exposures in comparison with existing exposure data $^{60}$. This comprehensive validation study has initially found relatively good initial correlation of the non-expert Stoffenmanager score with expert evaluation overall for inhalable dusts $\left(r_{\mathrm{s}}=0.83\right)$ and liquids $\left(\mathrm{r}_{\mathrm{s}}=0.81\right)$. This validation process for the Stoffenmanager model remains an ongoing process and is intended to remain a dynamic process with continual updating ${ }^{60}$.

Developed through the cooperation of corporations within the Norwegian oil industry, KjemiRisk is an assessment of chemical health risk based on experience and practice in these industries ${ }^{62}$. The tool takes the following into account: physical properties of the chemical, the handling of the chemical, and the appropriateness of the technical, organizational and personal barriers established to control the chemical exposure, and the duration and frequency of the work task using R- and safety phrases (S- phrases) as its basis. Similar to R-phrases, S-phrases are also required by the EU to appear on each label and safety data sheet for hazardous chemicals as part of the classification, packaging, and labeling of dangerous substances provision (Council Directive 67/548/EEC). Chemicals are grouped into one of five health hazard categories based on R-and S-phrases. As part of the KjemiRisk application, 15 common tasks are defined and the handling of the chemical, its physical state, duration and frequency of use, potential for exposure, and the appropriateness of controls in place are used in the conceptual model. The risk assessment is divided into two phases which include the potential risk and the final risk. These are adjusted for risk based on a judgment of the reliability and appropriateness of the established barriers and/or controls. The risk assessment provides a full risk evaluation of task-based work procedures based on an evaluation of risk for illness related to lungs, internal organs, and skin. KjemiRisk can be considered both a rough risk assessment tool when used by line managers or health and safety generalists and an expert tool when used by industrial hygienists. It is currently available in Norwegian and English as an individual or a network application when integrated with an appropriate server. Expansion of web applications, improvement of reporting functionalities, and substitution of capabilities are currently being considered for development ${ }^{62}$.

Developing and implementing CB risk assessment / management programs is critically important to many industries which process and market hazardous chemical substances. 
$\mathrm{CB}$ is an invaluable universal tool for assessing and managing these chemical risks. There is an important difference between industries which employ commodity industrial chemicals (e.g., bulk petro-chemical, health care, etc.) and those with unique proprietary chemicals (e.g., pharmaceutical, many industrial / commercial products, etc.). Many commodity industrial chemicals are well characterized, therefore, they can be assessed and controlled using existing CB models (COSHH Essentials, etc.). (Note that there is no need to use $\mathrm{CB}$ models to manage chemicals for which OELs exist.) However, industries which process and market unique proprietary chemicals must customize their risk assessment / management CB models for their operations using three essential steps: (1) performing appropriate hazard assessments to classify and communicate hazards; (2) assessing worker exposures in the workplace during specific operations; and (3) communicating, implementing, and verifying the proper control measures. Exposure Control Practices (ECPs) - specific guidance on the control measures - are a valuable tool for managing chemical risks. The ECPs provide a "feedback loop" to ensure that workers are protected and exposures are controlled to the desired levels. ECPs should be based on the Hierarchy of Control principles. Also, they must be verified as part of the exposure assessment program.. However, they enable much more robust risk assessment / management than do traditional IH approaches..

\section{Further Model Evolution}

Both the UK and ILO CB models focus on the use of bulk chemicals. In addition to chemical agents, which are covered by other UK regulations (i.e. asbestos, lead, and pesticides), they also are not intended to address process-generated emissions. These are chemical agent exposures created by the task, or not purchased in bulk, and include construction-related hazards such as silica dust, welding fume, and wood dust exposures as examples. Silica exposures in mining or construction have an excellent track record for existing interventions and practical solutions-based outcomes. These include standardized recommendations and subsequent reduction of exposures relating to the implementation of specified control solutions ${ }^{63,64,65}$. The UK HSE has already begun to adapt the CB model for broader chemical agents and expansion of the COSHH Essentials approach toward direct control advice. Exposures generated by these processes do not have Risk Phrases and require a different practical approach. The UK HSE has developed a CB process for some of these exposures by directing the user to job-specific control advice sheets relating to initial selected professions such as dry cleaning, hairdressing, and paint spraying ${ }^{40,66}$. Taking this a step further, the Silica Essentials is also directing users to control advice sheets that are industry and task-based and do not require the additional step of inputting data ${ }^{67}$. Instead the user selects the control advice directly by activity, avoiding the interim exposure prediction step of the COSHH Essentials model. The Silica Essentials is another CB model that is currently being evaluated in implementation and validation efforts internationally, including in Africa and Latin America.

The stratification of risk that began in the 1970s is now being considered for application in a variety of occupational health, hygiene, and safety professions as well in major industries. The international $\mathrm{CB}$ workshops have been an essential element in establishing uniform research agendas for evaluating $\mathrm{CB}$ strategies. They have also served to initiate the expansion of chemical-oriented models to best address practical prevention of a broader spectrum of work-related illness, disease, and injury. Topics discussed at these workshops that are beginning to be addressed include the provision of national-level guidance and coordination, pilot projects at the state level, and creation of an Occupational Risk Management (ORM) Toolbox. The ORM Toolbox approach is intended to broaden the $\mathrm{CB}$ model to include a more comprehensive exposure control 
basis for globally common industries such as construction and agriculture that require a multidisciplinary approach for chemical and ergonomic, safety, and environmental concerns. Current efforts have begun for the development of a $\mathrm{CB}$ model for a Construction Toolbox, addressing these composite, potential exposures by trade and $\operatorname{task}^{68}$. To achieve the ORM Toolbox approach a broader, multidisciplinary approach for trade-related exposures is needed.

Applying the CB model in a multidisciplinary fashion requires some brief consideration of differences between the fundamental approach to $\mathrm{IH}$, ergonomics, and occupational safety. Concepts on exposure and variability of exposure are well developed in the IH profession. These concepts are hardly present in occupational safety. Ergonomics and occupational safety both have a strong focus on design and redesign, which is much less developed in IH. Therefore, as CB models are being developed to address musculoskeletal disorders and occupational injuries, they may find professionals in these specialties well conditioned to this simplified adaptation. While CB strategies like the Silica Essentials are being developed to address locally generated exposures, as in the construction industry, the exposure factors relating to ergonomics are also being evaluated. Another IH to ergonomic comparison is that chemical production involves the development of new products which may never be fully researched and can logarithmically expand the variety of exposure routes and sources for a given worker. In contrast, ergonomics has a finite group of well-researched and defined risk factors and effective programs ${ }^{69,70}$. The ILO Ergonomics Checkpoints document is an example of well-researched and internationally validated models that is being developed as the basis of Ergonomics Toolkits ${ }^{71,72}$. Efforts in The Netherlands have begun to consider the incorporation of occupational safety requirements with a focus on traumatic injury ${ }^{73}$. Occupational safety is not restricted to chemical safety, but a more general approach is considered, focussing on causes of both major and minor occupational accidents. It has been presented that classifications already exist for various variables of accident causation, which can be viewed as an analogous 'banding principle' where safety phrases can be applied in a manner similar to risk phrases. In IH practice control of exposure takes place after the central event occurred, the emission of the hazardous substance. In occupational safety, barriers are active both before and after the central event. Therefore these barriers, including management factors, have a strong relation with the quality of safety management systems, and these factors are important parameters for risk prevention $^{73}$. The endpoint of this CB model would not necessarily lead to control advice as much as an identification and implementation of barriers. This barrier banding model would apply these phrases to provide information on the type of hazard of accident scenarios or related situations and will guide the type of precautions needed deal with these scenarios or situations ${ }^{74}$.

Moving back to the roots of the modern $\mathrm{CB}$ movement, nanotechnology industries are also finding a limitation in toxicological data in a manner similar to their biological and pharmaceutical counterparts. They also have to achieve a risk management program with an insufficient basis for traditional IH quantitative risk assessment approaches. An important distinction is that they have a longer track record of CB models to work with in developing a control approach. To develop the concept, Maynard ${ }^{10}$ has combined the proven effectiveness of $\mathrm{CB}$ in controlling exposures in an intensive research and development industry, such as in pharmaceuticals, with the utility of COSHH Essentials model. A conceptual CB model is presented which offers the same four control approaches of the UK model as stratified by corresponding 'impact' and exposure indices. This model proposes combining engineered nanomaterial composition 
parameters such as shape, size, surface area, and surface activity with their exposure availability in terms of dustiness and amount in use and linking these indices to bands with corresponding control approaches. This nanomaterial $\mathrm{CB}$ model, although not developed in practice, is presented similarly to COSHH Essentials in that it is a useful concept that affords a pragmatic approach to exposure control and is considered to be an alternative rather than a substitute to traditional IH risk assessment and control ${ }^{10}$.

\section{DISCUSSION}

Underpinning the toxicological basis of the UK approach is the importance of an accurate toxicological rating and hazard band classification by suppliers of chemical substances. Given this critical need for CB models, there is a need to reevaluate the assignment of Rphrases to chemical substances ${ }^{26,42}$. This process should go beyond work with the COSHH Essentials model and become a central focus for the different CB models available and in development. Significant concerns have been raised about the accuracy of EU classifications of chemical substances ${ }^{42}$. If COSHH Essentials has been designed to be slightly over-protective ${ }^{25}$, then the $15 \%$ of evaluated EU classifications that were assigned too low a danger class ${ }^{42}$ should be considered a substantial issue to be addressed. Other confounding issues for the model also require further evaluation. The margins of safety are possibly inadequate for many vapors and some may need to be classified into higher hazard bands ${ }^{26,31,37}$. There is also a variation in hazard band assignment between the COSHH Essentials model and ILO Toolkit ${ }^{31,37}$. In addition to model validation efforts, experts who have written on the CB topic confirm its potential value as a risk assessment and risk management tool in the workplace. They also express caution about the need for systematic, critical evaluation of the approach before widespread adoption.

According to Money ${ }^{13}$, "no systematic evaluation of the actual impact and effectiveness of the schemes has been undertaken ... no systematic assessment has been undertaken of the impact that $\mathrm{CB}$ approaches have had on the management of risk at the workplace or other levels. Thus, in terms of future developments in the area, it would appear that before further refinements are considered, there needs to be an extensive and systematic evaluation of the uptake and impact of a number of the key approaches." Swuste et al. ${ }^{11}$ referenced Kromhout ${ }^{35}$, stating that "The COSHH Essentials has met some criticism in the literature, focusing on the lack of a proper evaluation before its introduction into the occupational arena, as well as the generic nature of the tool, which will lack precision and accuracy in situations where these are required." Tischer and his colleagues ${ }^{32}$ have said that in the German occupational hygiene community, "...there was consensus that the scheme [COSHH Essentials] had great potential for further development. On the other hand, with respect to the exposure predictive model it has been argued that, due to its generic character, reliability and accuracy (safety) may have been sacrificed for the sake of simplicity and transparency. However, this assumption is not based on real measurement data and instead reflects the low degree of confidence generally enjoyed by generic models."

The impetus for the modern movement of $\mathrm{CB}$ was the regulatory driven need to address chemical exposures for the majority of the UK workforce. COSHH Essentials, the UK model, was created by experts who, with much thought, chose a simplified model to achieve maximum utility in addressing this need. The work of Cherrie and Schneider ${ }^{24}$ served to strengthen this decision by showing that a structured approach based on descriptive workplace activities provided significant correlation with exposure 
measurements. The dissection and examination of this $\mathrm{CB}$ model remains an ongoing endeavor. However, its effectiveness in achieving its intended utility is often overlooked as a prime component. Results from HSE survey on the use and application of the COSHH Essentials model ${ }^{43}$ infer utility in the UK. This CB model, however, is also being considered for use in many other countries around the world, including the US. For this reason the COSHH Essentials model has received additional attention and should receive more to ensure an ongoing critical evaluation to determine whether the model delivers target exposure ranges, offers controls commensurate to exposure potential, is used appropriately, and has improved control of exposure.

In consideration for its implementation, Oldershaw ${ }^{75}$ cautioned that the COSHH Essentials approach cannot be adopted uncritically by other countries; further, the approach must be seen in the context of personal protection, training, health surveillance as appropriate, etc. A key point is that the approach is not meant to replace exposure measurement, interpretation, substance, and chemical control. These studies and expert comments presented in the literature are not "project stoppers," but rather emphasize the need for collection of data under controlled scenarios to validate the predictions of the model. Under-prescription of control could lead to serious injury, while over-prescription could lead to significant unnecessary expense, especially for SMEs. Of the two types of error, i.e., under-control (recommendation of inadequate level of control) is potentially more serious than over-control. In this model's development the general approach was to be conservative or slightly over-protective ${ }^{25}$.

Internal evaluations of the UK model have shown under-control error for small-scale, dispersed use of solvents and some powder handling operations ${ }^{9,32}$ as well as for vapor degreasing and bag filling operations ${ }^{41}$. These results seem to confirm Kromhout's argument $^{33}$ that potential misclassification of exposure bands can consequently affect assignment to control bands. Brooke's work predicted this potential; however, this concern was essentially addressed with expectations that the model's scheme and the allocation of hazard bands with R-phrases would be consistently evaluated and improved $^{26}$, however the research has not shown this to date. However, with external evaluation the COSHH Essentials model has also been found to deliver a significant level of confidence in the target exposure ranges ${ }^{32}$. German BAuA comparisons of the model's outcomes compared to personal exposure monitoring data, in a number of different industries, were well within range for work with solids and medium scale liquids ${ }^{32,39}$, although some under-control error with liquids was found in their work ${ }^{39}$ as well as Brooke's ${ }^{26}$. The ILO Chemical Toolkit, based on COSHH Essentials, has also been shown to indicate more conservative control solutions based on comparisons with the Singapore's SQRA method utilizing personal exposure monitoring data for deriving risk level approaches ${ }^{57}$. Comparisons indicate that, for the majority of the 27 processes selected, the Toolkit equally- or over-evaluated the risk relative to the SQRA ${ }^{57}$. Within these validation efforts there has been an acknowledged paucity of data with which to validate CB models $3,11,13,32,33,41$. There is also a limited range of exposure situations with which to compare predictions ${ }^{32}$. There has also been difficulty in ascertaining reported control classification ${ }^{3}$, proper characterization of specific work parameters, and materials in use $^{41}$ for comparison of predicted and actual exposures. The need for health surveillance data / environmental monitoring must be evaluated ${ }^{25}$; particularly when toxicological data are limited ${ }^{20}$. The ongoing need for personal monitoring (air and wipe tests) must be strongly emphasized. The use of the CB models is to complement, not replace the traditional $\mathrm{IH}$ approach to risk and exposure assessment. Therefore, personal monitoring is needed to bolster a system that evaluates the effectiveness of controls 
initially and over time. It will continue to be an essential requirement that ongoing monitoring is needed to detect breaches in containment systems and effectiveness of LEV, even if previously verified ${ }^{5}$.

The work of Jones and Nicas ${ }^{37,41}$ has received much attention as its critique of the COSHH Essentials and ILO Toolkit have indicated a high prevalence of control errors ${ }^{41}$ and the potential for an inappropriate confidence in the workplace chemical exposure reduction $^{37}$. HSE members responded to their COSHH Essentials evaluation ${ }^{41}$ clarifying that their CB model is not intended to predict exposure, but rather to identify adequate control approaches ${ }^{76}$. This is a difficult statement to justify in that the exposure prediction step is what separates the COSHH Essentials model from the earlier toxicology-tocontrol pharmaceutical CB model. It was also indicated that the article ${ }^{41}$ did not actually evaluate the COSHH Essentials as, of the workplace exposures utilized, none of the controls in place were recommended by their CB model. Non-HSE members also responded $^{77}$ to these Jones and Nicas articles, noting that the intent of COSHH Essentials is its utility in obtaining and implementing appropriate risk control advice and that user evaluation trials have indicated a higher likelihood of achieving this than if presented in a less accessible or understandable format. Jones and Nicas replied to this commentary ${ }^{78}$ indicating that without a recommended prospective study of COSHH Essentials, evaluation of its components is necessary. While confirming their approach and remaining skepticism of the model's outcomes, they do address their study's limitations in that the variability of engineering control efficiency may also be seen in the high rate of under-control findings. Their margin of safety applications in their assessment of the ILO Toolkit ${ }^{37}$ also requires evaluation. Their reliance on safety margins may not be appropriate for validation studies in that their conclusions are heavily dependent on the critical effect's relative toxicity. Higher consequence toxicological outcomes such as cancer require a much larger safety margin than for lower outcomes such as irritation, and may therefore affect the probability of an under-control finding with more adverse toxicological outcomes.

An important distinction in the development of the UK model is that the current objective of the COSHH Essentials is to achieve exposure levels anywhere in the exposure band, whereas the CIA recommends that exposures should be maintained "as low as reasonably practicable"19,20. This disconnect with the trade association should be further investigated. Although comparisons to solid chemical exposures have been promising, model validation efforts have shown that it is difficult for researchers to retrospectively evaluate the dustiness of particulates and it may therefore be difficult for SME managers to do the same $^{41}$. For liquid chemical exposures, under-control error in small-scale solvent applications, although consistent with Brooke's ${ }^{26}$ reservations on vapor's equivalency with dusts as in Table III, can in part be attributed to industrial tasks that spread relatively minute quantities over a large surface area, increasing exposure potential. An adjustment or acknowledgement within the control guidance sheets can be made for tasks with these processes, however the model's weakness with vapors must be further evaluated. In addition, the Regetox approach ${ }^{12}$ presented composition by weight for both liquid and solid mixtures in evaluating risks for each harmful component in a workshop that prepares plasticizing mix. Composition by weight is appropriate for solids, but this may skew the estimation of potential risk for liquids as composition should be by molar fraction due to the difference in volatility of various liquid components.

Promising information is just beginning to be put forth in the evaluation of The Netherlands' Stoffenmanager CB model. Their approach has benefited from the ongoing 
critique of the COSHH Essentials and ILO Chemical Control Toolkit which has assisted in their decision to utilize exposure assessment prioritization in its banding strategy which has been derived from the international validation process which include the international CB workshops ${ }^{60,61}$. Stoffenmanager serves as an excellent example of how dissecting existing models can lead to criteria to be used in developing other exposure control models. Its initial validation study remains an ongoing process, but preliminary information shows that the current generic version of Stoffenmanager indicates its utility as an exposure assessment tool for SME managers and may be an appropriate CB model for use in Tier 1 scenarios relating to $\mathrm{REACH}$. Future efforts include an English version of the generic model in late 2007, creating opportunities for wider international use and further validation of the model and verification of the effectiveness of its control outcomes. Also in progress is an expansion of this $\mathrm{CB}$ model into branch specific versions that is expected to become a standard in The Netherlands, and the development of a dynamic web-based data exchange module called STEAMBASE (SToffenmanager Exposure And Modeling dataBASE) ${ }^{60}$ which may be an important foundation for the prospective studies that are a consensus in CB literature.

Regulatory requirements in the UK were a driver to develop the COSHH Essentials CB model for non-experts to address exposure to chemicals. The model was simplified by design due to the many SME managers under this regulation who do not have easy or affordable access to professional judgment. The pharmaceutical and biological agent exposure control models, the evolutionary predecessors of the modern CB movement, were and are intended for use in Large Enterprises (LE). Due to their size, these industries typically have adequate access to professional expertise and funding for engineering controls and their maintenance. Models relating to pharmaceutical agents, as an example, can therefore be more intricate and achieve greater accuracy as they are implemented and maintained by trained professionals. The lack of toxicological data and availability of established OELs are the common bases for the creation of both the pharmaceutical agent and chemical exposure control models.

The developers of the COSHH Essentials and the related ILO Chemical Control Toolkit both deliberately chose a less complex model in order to achieve simplicity. The ease-ofuse of the UK model has been for the most part achieved for the intent of its development in the UK -- use and application by SME managers. However, a key distinction between the models is that the ILO developed its international version for use by non-experts everywhere in the world. This expertise may not available due to limited funds, such as in the EU or the US, or due to the relative absence of the IH profession in most industrially developing nations worldwide, affecting LEs as well as SMEs ${ }^{79}$. It was understood in the development of the modern CB models that a practical exposure control tool for nonexperts may in practice compromise a level of accuracy when compared to the advice of experts. As important as this is to achieve utility for the intended audience -- whether for SMEs, developing countries, or for experts and non-experts alike in the absence of OELs -- validation of these models has indeed pointed out areas where this accuracy has been compromised. The focal point then becomes one of perceived risk and the variable levels of acceptability of risk, a perception that varies from country to country, from culture to culture.

The historical basis for the modern CB models was that they were to be used by experts within a research and development environment. The need for this approach was primarily related to the absence of OELs, such as in the biological, pharmaceutical, and now the nanotechnology industries. Validation of these models is complicated in that 
traditional exposure assessment may not be possible at this time without a proven toxicological basis, as is especially apparent with nanoparticulate ${ }^{10}$. What all these $\mathrm{CB}$ models have in common is achieving a level of approachability to what otherwise may remain only in the hands of those with access to expert judgment. They also share a certain acceptance of risk and inaccuracy. Adaptation of the existing models beyond bulk chemical use has been assisted by this cumulative $\mathrm{CB}$ discussion in that developers can learn from still ongoing evaluations and benefit from a growing acceptability of simplicity in achieving exposure reduction. The practical nature of the silica, ergonomics, and injury prevention CB model approaches indicates that they are likely to succeed; however, not without the same rigor of validation and evaluation that should be given to all CB models. In developing multidisciplinary CB strategies it has become apparent that involvement of stakeholders is helpful in defining minimum performance standards, whether required by regulation or by circumstance.

\section{CONCLUSION}

Further research remains a requirement for all $\mathrm{CB}$ models. This includes further internal validation of $\mathrm{CB}$ model components, broader external validation of the model predictions when compared to expert interventions, and especially the need for operational analysis of the model as implemented to achieve intended outcomes. A prospective research process therefore remains essential to achieve an understanding of the implications of the model as applied and how this correlates to its overall effectiveness for its target group. This will assist in addressing the remaining questions as to how control recommendations are being implemented and maintained and whether they are achieving the intended exposure reduction. The lack of this information has led many to question the overall effectiveness of $\mathrm{CB}$ models in that they have knowingly chosen simplicity at the expense of accuracy and, therefore, protection of the worker. This research needs to be performed and the results folded into an improvement process for $\mathrm{CB}$ models, which must include continual reevaluation of R-phrases and GHS Hazard Statements, in order to scientifically address these questions. In addition, further field studies are also vital to this research as they are necessary for providing essential validation and verification data which in turn will improve our practical understanding of the strengths and weaknesses of each of the models. In the absence of this information, the CB models as currently available are best used when OELs do not exist or as initial risk assessment screening tools that at some level include expert input and traditional IH monitoring.

It seems that lost in these scientific validation discussions are the billions of workers who do not have access to expert advice. When further research is performed it must not stop short at the dissection of models. It must use the lessons learned from the process to build a better model that does have a place in the hands of non-experts. CB models are therefore, in essence, an opportunity to simplify the best of scientific information into a format that is accessible to the multitudes. Expert IH advice in practice is expensive and is non-existent in many countries, rendering it inaccessible to so many. This fact should not be used as an excuse to apply unvalidated control models blindly, but rather to serve as an impetus to expand the reach of this expertise and to develop it where it does not exist. With this in mind, the modern CB movement should continue to seek the finest technical expertise to make the models as good as possible. Seeking perfection will only ensure that the prevention of work-related disorders will not be achieved for the majority of the world's workforce. 


\section{ACRONYM GLOSSARY}

\begin{tabular}{|c|c|}
\hline ABPI & Association of the British Pharmaceutical Industry \\
\hline BAuA & Federal Institution for Industrial Safety and Medicine (Germany) \\
\hline BOHS & British Occupational Hygiene Society \\
\hline $\mathbf{C B}$ & Control Banding \\
\hline CEFIC & European Chemical Industry Council \\
\hline CEMAS & CEFIC Exposure Management System \\
\hline CGS & Control Guidance Sheets \\
\hline CIA & Chemical Industries Association \\
\hline CHIP & Chemical Hazardous Information and Packaging \\
\hline COSHH & Control of Substances Hazardous to Health \\
\hline EASE & Estimation and Assessment of Substances Exposure \\
\hline ECETOC & European Centre for Ecotoxicology and Toxicology of Chemicals \\
\hline ECHA & European Chemical Agency \\
\hline ECP & Exposure Control Practices \\
\hline EINECS & European Inventory of Existing Substances \\
\hline $\mathbf{E U}$ & European Union \\
\hline GHS & Globally Harmonized System of Classification and Labeling of Chemicals \\
\hline GTZ & German Technical Cooperation (German) \\
\hline HHE & Health Hazard Evaluation \\
\hline HSE & Health and Safety Executive (UK) \\
\hline HSDB & Hazardous Substances Data Base \\
\hline ICCT & International Chemical Control Toolkit (new name of the ILO Toolkit) \\
\hline IH & Industrial Hygiene \\
\hline ILO & International Labor Organization \\
\hline IOHA & International Occupational Hygiene Association \\
\hline $\mathbf{L E}$ & Large Enterprises \\
\hline LEV & Local Exhaust Ventilation \\
\hline LOAEL & Lowest Observed Adverse Effect Level \\
\hline МАK & Maximum Allowable Concentrations (Germany) \\
\hline MoE & Margins of Exposure \\
\hline NIOSH & National Institute for Occupational Safety and Health (US) \\
\hline NOAEL & No Observed Adverse Effect Level \\
\hline OEB & Occupational Exposure Band \\
\hline OEL & Occupational Exposure Limit \\
\hline ORM & Occupational Risk Management \\
\hline PACE & Prevention and Control Exchange \\
\hline PB-ECL & Performance-Based Exposure Control Limits \\
\hline PPE & Personal Protection Equipment \\
\hline PPM & Parts per million \\
\hline REACH & Registration, Evaluation, and Authorization of Chemicals \\
\hline RTECS & Registry of Toxic Effects of Chemical Substances \\
\hline SME & Small- and Medium-Sized Enterprises \\
\hline SQRA & Semi-Quantitative Risk Assessment \\
\hline $\mathbf{T L V}^{\circledR}$ & Threshold Limit Value \\
\hline TWA & Time Weighted Average \\
\hline UK & United Kingdom \\
\hline US & United States \\
\hline WHO & World Health Organization \\
\hline
\end{tabular}




\section{REFERENCES}

1. Garrod, A. and R. Rajan-Sithamparananadarajah: Developing COSHH essentials; dermal exposure, personal protective equipment and first aid. Ann. Occup. Hyg. 47:577588 (2003).

2. Jackson, H.: Control Banding - Practical tools for controlling exposure to chemicals. Asian-Pacific Newsletter 9:62-63 (2002).

3. Maidment, S.C.: Occupational hygiene considerations in the development of a structured approach to select chemical control strategies. Ann. Occup. Hyg. 42:391-400 (1998).

4. Sargent, E.V. and G.D. Kirk: Establishing airborne exposure control limits in the pharmaceutical industry. Am. Ind. Hyg. Assoc. J. 49:309-313(1998).

5. Naumann, B.D., E.V. Sargent, B.S. Starkman, W.J. Fraser, G.T. Becker, and G.D. Kirk: Performance-based exposure control limits for pharmaceutical active ingredients. Am. Ind. Hyg. Assoc. J. 57: 33-42 (1996).

6. Farris, J. P., A.W. Ader, and R.H. Ku: History, implementation, and evolution of the pharmaceutical hazard categorization and control system. Chemistry Today 24:5-10 (2006).

7. Tait, K.: Control banding: an improved means of assessing and managing health and safety risks at Pfizer. Presented at the Second International Control Banding Workshop (2ICBW), 1-2 March 2004, Cincinnati, OH. (2004).

8. Centers for Disease Control and Prevention: Biosafety in Microbiological and Biomedical Laboratories. $3^{\text {rd }}$ Rev. J.Y. Richmond and R.W. McKinney eds. [HHS pub. No. (CDC) 93-8395] Washington, DC: US Government Printing Office 1993. pp. 6-43, 138-145.

9. Association of the British Pharmaceutical Industry (ABPI): Guidance on setting inhouse occupational exposure limits for airborne therapeutic substances and their intermediates. London: ABPI (1995).

10. Maynard, A.: Nanotechnology; the next big thing or much ado about nothing? Ann. Occup. Hyg. 51: 1-12 (2007).

11. Swuste, P., A. Hale, and S. Pantry: Solbase: a databank of solutions for occupational hazards and risks. Ann. Occup. Hyg. 47:541-547 (2003).

12. Balsat, A., J. de Graeve, and P. Mairiaux: A structured strategy for assessing chemical risks, suitable for small and medium-sized enterprises. Ann. Occup. Hyg. 47: 549-56 (2003).

13. Money, C.D.: European experiences in the development of approaches for the successful control of workplace health risks. Ann. Occup. Hyg. 47:533-540 (2003). 
14. Money, C. D.: A structured approach to occupational hygiene in the design and operation of fine chemical plant. Ann. Occup. Hyg. 36:601-607 (1992).

15. Henry, B.J. and K.L. Schaper: PPG's safety and health index system: a 10-year update of an in-plant hazardous materials identification system and its relationship to finished product labelling, industrial hygiene, and medical programs. Am. Ind. Hyg. Assoc. J. 51:475-484 (1990).

16. Gardner, R.J. and P.J. Oldershaw: Development of pragmatic exposure-control concentrations based on packaging regulation risk phrases. Ann. Occup. Hyg. 35:51-59 (1991).

17. Woodward, K.N., A. McDonald, and S. Joshi: Ranking of chemicals for carcinogenic potency--a comparative study of 13 carcinogenic chemicals and an examination of some of the issues involved. Carcinogenesis 12:1061-1066 (1991).

18. Crabtree, H.C., D. Hart, M.C. Thomas, B.H. Witham, I.G. McKenzie, and C.P. Smith: Carcinogenic ranking of aromatic amine and nitro compounds. Mutation Res. 264:155-162 (1991).

19. Chemical Industries Association (CIA): Safe handling of colourants 2 . London: CIA (1993).

20. Guest, I.: The Chemical Industries Association Guidance on Allocating Occupational Exposure Bands. Ann. Occup. Hyg. 42:407-411 (1998).

21. European inventory of existing commercial chemical substances (EINECS): EINECS Vols. I - VIII. Commission of the European Communities, EEC, Brussels, Belgium (1987).

22. CIA: The control of substances hazardous to health regulations: guidance on allocating occupational exposure bands. (Regulation 7). London: CIA (1997).

23. Burstyn, I. and K. Teschke: Studying the determinants of exposure: a review of methods. Am. Ind. Hyg. Assoc. J. 60:57-72 (1999).

24. Cherrie, J. and T. Schneider: Validation of a new method for structured subjective assessment of past concentrations. Ann. Occup. Hyg. 43:235-245 (1999).

25. Russell, R.M., S.C. Maidment, I. Brooke, and M.D. Topping: An introduction to a UK scheme to help small firms control health risks from chemicals. Ann. Occup. Hyg. 42:367-376 (1998).

26. Brooke, I.M.: A UK scheme to help small firms control health risks from chemicals: toxicological considerations. Ann. Occup. Hyg. 42:377-390 (1998).

27. Royal Society of Chemistry (RSC): COSHH in laboratories, 2nd edition. London: RSC (1996).

28. CIA: Safe handling of potentially carcinogenic aromatic amines and nitrocompounds. London: CIA (1992). 
29. Oldershaw, P.: Control banding - a practical approach to judging control methods for chemicals. J. Prev. Med. 9:52-58 (2001).

30. Hudspith, B. and A.W. Hay: Information needs of workers. Ann. Occup. Hyg. 42:401-406 (1998).

31. Jones, R.M. and M. Nicas: Evaluation of the ILO Toolkit with regards to hazard classification and control effectiveness." Presented at 2ICBW, 1-2 March 2004, Cincinnati, OH. (2004).

32. Tischer, M., S. Bredendiek-Kamper, and U. Poppek: Evaluation of the HSE $\mathrm{COSHH}$ Essentials exposure predictive model on the basis of BAuA field studies and existing substances exposure data. Ann. Occup. Hyg. 47:557-569 (2003).

33. Kromhout, H.: Design of measurement strategies for workplace exposures. Occup. Environ. Med. 59:349-354 (2002).

34. Topping, M.: Design of measurement strategies for workplace exposures: Letter to the editor." Occup. Environ. Med. 59:788 (2002).

35. Kromhout, H.: Author's reply. Occup. Environ. Med. 59:788-789 (2002).

36. Topping, M.: COSHH Essentials from concept to one stop system." Presented at the First International Control Banding Workshop, 4-5 November 2002, London, UK (2002).

37. Jones, R.M. and Nicas M.: Margins of Safety Provided by COSHH Essentials and the ILO Chemical Control Toolkit. Ann. Occup. Hyg. 50:149-156 (2006).

38. Jayjock, M.A., J.R. Lynch, and D.I. Nelson: Fundamentals of Risk Assessment for Practicing Industrial Hygienists. Fairfax, VA, American Industrial Hygiene Association (2000).

39. Tischer, M.: Current BAuA/GTZ research on occupational exposure and control strategies: recent results from various industrial areas and from Indonesian SMEs. Presented at the First International Control Banding Workshop (IICBW), 4-5 November 2002, London, UK (2002).

40. Tischer, M.: Helping SMEs to manage risks from chemicals in the workplace. Proceedings from an international workshop, 26-27 November 2001, Amsterdam, The Netherlands. Zeist: TNO Nutrition and Food Research (2001).

41. Jones, R.M. and Nicas M.: Evaluation of COSHH Essentials for Vapor Degreasing and Bag Filling Operations. Ann. Occup. Hyg. 50:137-147 (2006).

42. Ruden, C. and S.O. Hansson: How accurate are the European Union's classifications of chemical substances. Toxicol. Lett. 144:159-172 (2003).

43. Evans, P.: COSHH Essentials. Presented at the National Control Banding Workshop, 9-10 March 2005, Washington, DC (2005). 
44. European Commission (EC): Council Directive 98/24/EC on the protection of the health and safety of workers from risks related to chemical agents at work. Off. $J$. Eur. Commun. L131 (1998).

45. Chemical Industry Union (UIC): [Tool for evaluation of the risks to the chemicals], [Document in French] technical document DT 63. Paris: UIC (1999).

46. European Chemicals Agency. REACH. Available at http://ec.europa.eu/environment/chemicals/reach/reach intro.htm. Accessed 15 June 2007.

47. Money, C.D.: Data solutions for the 21st century: CEFIC's vision and intentions. The European Chemical Industry Council. Appl. Occup. Environ. Hyg. 16:329-330 (2001).

48. European Centre for Ecotoxicology and Toxicology of Chemicals (ECETOC): Outline concept of a pragmatic approach to risk assessment. Brussels: ECETOC (2002). http://www.ecetoc.org.

49. Health and Safety Executive (HSE): 100 Practical applications of noise reduction methods. HSE. HMSO. London (1993).

50. Mitchel, T., Else, D.: Noise control in mining: seventy-five noise control solutions. Victorian Institute of Occupational Safety and Health, Ballarat University College, Victoria, Australia (1993).

51. Swuste, P. and A. Hale: Databases on measures to prevent occupational exposure to toxic substances." Appl. Occup. Environ. Hyg. 9:57-61 (1994).

52. Swuste, P., M. Corn, and B. Goelzer: Hazard prevention and control in the work environment; Report of a WHO meeting. African Newsletter 1:20-21 (1995).

53. Silk J.: The Globally Harmonized System (GHS) of Classification and Labeling of Chemicals: Harmonized Phrases for International Control Banding. Presented at 2ICBW, 1-2 March 2004, Cincinnati, OH. (2004).

54. Balsat, A., P. Mairiaux, and J. De Graeve: A global approach for assessing and managing chemical risks at the workplace: Advantages, limits and perspectives.

Presented at 1ICBW, London, 4-5 November (2002).

55. Balsat, A., P. Mairiaux, and J. de Graeve: Control banding for assessing chemical risks Belgian companies experience. Presented at 2ICBW, 1-2 March 2004, Cincinnati, OH. (2004).

56. Vincent, R. and F. Bonthoux: [Evaluation of chemical risk : hierarchisation of "risk potential".] [Article in French] I Cahiers de notes documentaires - Hygiene et securite du travail 179:29-34 (2000).

57. Yap, S.M.: Assessing the utility of the ILO Toolkit in Singapore. Presented at 2ICBW, 1-2 March 2004, Cincinnati, OH. (2004). 
58. Adelmann, K. Chemical Safety and Development. Society for Technical Cooperation (GTZ) [German], 1-10 (2001).

59. Tischer, M. and S. Scholaen: Chemical management and control strategies: experiences from the GTZ pilot project on chemical safety in Indonesian small and medium-sized enterprises. Ann. Occup. Hyg. 47:571-575 (2003).

60. Tielemans, E.: Evaluation of the Stoffenmanager. Presented at the Occupational Hygiene Conference, 17-19 April, Glasgow (2007).

61. Tijssen, S., M. le Feber, H. Heussen, J. West, and D. Noy: A new tool for small and medium enterprises to work safely with hazardous substances. Presented at $2 I C B W$, 1-2 March 2004, Cincinnati, OH. (2004).

62. Smedbold, H.T.: KjemiRisk - The Norwegian offshore approach to control banding. Presented 2ICBW, 1-2 March 2004, Cincinnati, OH. (2004).

63. Goldsmith, D.F.: The link between silica dust levels, risk assessments, and regulations. J. Expo. Anal. Environ. Epidemiol. 7:385-395 (1997).

64. Flynn, M.R. and P. Susi: Engineering controls for selected silica and dust exposures in the construction industry--a review. Appl. Occup. Environ. Hyg. 18:268-277 (2003).

65. Rappaport, S.M., M. Goldberg, P. Susi, and R.F. Herrick: Excessive Exposure to Silica in the US Construction Industry. Ann. Occup. Hyg. 47:111-122 (2003).

66. Evans, P.: Silica Essentials and the Chemical Control Toolkit. Presented at the Third International Control Banding Workshop (3ICBW), 21 September 2005, Pilanesberg, South Africa. (2005).

67. Evans, P.: COSHH Essentials - Reducing Silicosis. Presented at 3ICBW, 21 September 2005, Pilanesberg, South Africa. (2005).

68. Spee, T.: Risk assessment from toxic substances and control measures in the Dutch construction industry. Presented at 3ICBW, 21 September 2005, Pilanesberg, South Africa. (2005).

69. Stewart, K., M. Kiefer, P. Johnson, and D.M. Zalk: Establishing Ergonomics in Industrially Developing Countries. Proceedings SS (K1), International Occupational Hygiene Scientific Conference, 21 September 2005, Pilanesberg, South Africa. (2005).

70. Zalk, D.M.: Grassroots ergonomics: initiating an ergonomics program utilising participatory techniques. Ann. Occup. Hyg. 45:283-289 (2001).

71. Zalk, D.M.: Control banding's human health-based risk management strategies. Presentation at American Industrial Hygiene Conference Roundtable 221, Chicago, IL (2006). 
72. Zalk, D.M.: Control banding principles to reduce musculoskeletal disorders; the ergonomics toolkit." Proceedings V5(327), International Ergonomics Association Congress, Seoul, South Korea, (2003)..

73. Swuste, P.: Control banding, expansion of range; safety. Presented at 3ICBW, 21 September 2005, Pilanesberg, South Africa. (2005).

74. Zalk, D.M.: Practical prevention in safety; from control banding to barrier banding. Proceedings 17(5), International Working on Safety Conference, 15 September 2006 Eemhof, The Netherlands (2006).

75. Oldershaw, P.J.: Control banding workshop, 4-5 November 2002, London. Ann Occup Hyg 47:531-532 (2003).

76. Evans, P. and A. Garrod: Evaluation of COSHH Essentials for vapour degreasing and bag-filling operations. Letter to the Editor. Ann. Occup. Hyg. 50:641 (2006).

77. Money, C., S. Bailey, M. Smith, A. Hay, B. Hudspith, D. Tolley, et al.: Evaluation of the utility and reliability of COSHH Essentials. Letter to the Editor. Ann. Occup. Hyg. 50:642-643 (2006).

78. Jones, R.M. and Nicas M.: Author's reply. Ann. Occup. Hyg. 50: 643-644 (2006).

79. Eijkemans, G. and I. Fedetov: Global implementation strategy of the occupational risk management toolbox (control banding). Presented at $3 I C B W, 21$ September 2005, Pilanesberg, South Africa. (2005).

\section{Proceedings}

- First International Control Banding Workshop (1ICBW)

- $\quad$ http://www.bohs.org/eventDetails.aspx?event=42 Accessed 14 October 2007.

$\bullet$

- Second International Control Banding Workshop (2ICBW)

- $\quad$ http://www.acgih.org/events/ControlBand Accessed 14 October 2007.

$\bullet$

- Third International Control Banding Workshop (3ICBW) http://www.saioh.org/ioha2005/Proceedings/SSI.htm Accessed 14 October 2007.

\section{ACKNOWLEDGEMENTS}

We would like to thank all those who have assisted and the organizations that have supported all aspects of the International Control Banding Workshops, whose efforts have assisted in developing and driving the CB research agenda. Special thanks to Paul Swuste for his keen insight and direction in the development of this manuscript. Thanks also to Henri Heussen for his timely update on the Stoffenmanager validation process. Note; *Work performed in part under the auspices of the US Department of Energy by UC, Lawrence Livermore National Laboratory under contract W-7405-Eng-48. 